\title{
Du rat-toto fils du porc-épic, de la panthère et de la nandinie
}

Savoir encyclopédique et pensée analogique sur l'origine des masques de la famille Zamble en pays Gouro (RCI)

Gambian rat the son of porcupine, panther and African palm civet: encyclopedic knowledge and analogical thinking about the origin of Zamble family masks in Guro country (RCI)

\section{Claudie Haxaire}

\section{(2) OpenEdition}

\section{Journals}

Édition électronique

URL : http://journals.openedition.org/ethnoecologie/6216

DOI : 10.4000/ethnoecologie.6216

ISSN : 2267-2419

Éditeur

Laboratoire Eco-anthropologie et Ethnobiologie

Référence électronique

Claudie Haxaire, "Du rat-toto fils du porc-épic, de la panthère et de la nandinie », Revue d'ethnoécologie [En ligne], 18 | 2020, mis en ligne le 31 décembre 2020, consulté le 29 janvier 2021. URL : http://

journals.openedition.org/ethnoecologie/6216; DOI : https://doi.org/10.4000/ethnoecologie.6216

Ce document a été généré automatiquement le 29 janvier 2021.

\section{(c) $(1)$}

Revue d'ethnoécologie est mis à disposition selon les termes de la licence Creative Commons Attribution - Pas d'Utilisation Commerciale - Pas de Modification 4.0 International. 


\section{Du rat-toto fils du porc-épic, de la panthère et de la nandinie}

Savoir encyclopédique et pensée analogique sur l'origine des masques de la famille Zamble en pays Gouro (RCI)

Gambian rat the son of porcupine, panther and African palm civet: encyclopedic knowledge and analogical thinking about the origin of Zamble family masks in Guro country (RCI)

\section{Claudie Haxaire}

\section{NOTE DE L'AUTEUR}

Les identifications animales ont été vérifiées par l'ethno-ornithologue Koué bi Mathieu, que je remercie.

1 Nous proposons une balade ${ }^{1}$ naturaliste parmi les chants des mascarades Gouro de la famille Zamble, masques internationalement connus ${ }^{2}$. Naturaliste, parce que ce sont bien leurs surnoms animaux et leurs filiations, ou leurs attributs végétaux, et donc les mœurs et propriétés assignées à ces masques, qui révèlent la nature de leurs personnages. Et parce que seul le savoir encyclopédique transmis par nos hôtes autorise cette riche et poétique dérive dans une pensée analogique malheureusement parfaitement négligée des amateurs d'art. Nous nous laisserons guider par les chants où les personnages prennent paradoxalement d'autres genres, le beau Zamble, posté telle la panthère $\mathrm{e}^{3}$ sur une termitière bien propre, a pour frère ou mari le vilain Zàùli, aux joues gonflées de kola ${ }^{4}$, fille du rat-toto ${ }^{5}$, lui-même fils du porc-épic ${ }^{6}$, lequel est bien l'un des fétiches du masque du bois sacré je qui, lui, ne sort que la nuit, loin des regards profanes. Leur épouse commune ${ }^{7}$ Gù (ton bas), ne tiendrait-elle pas de la nandinie ${ }^{8} g u$ (ton moyen) fils du père des puissants animaux qui dévorent leurs congénères?

2 Le masque Zamble, cité par l'auteur de la première monographie sur les Gouro (Tauxier 1924), semble avoir déjà existé au début du XIX ${ }^{e}$ siècle. On désigne couramment sous ce 
nom générique une famille de trois masques Zàùlì (tons bas), Zamble et Gù. Tous les masques sont nommés $y u$ " puissance »; en effet yu désigne tout support de jale, "la force vitale » que chacun détient à des degrés variables (Haxaire 1998); ces masques en sont éminemment dotés 9 grâce aux sacrifices dont ils sont destinataires. Zàùlì, Zamble et Gù sont dits plo-ji-yu «masques de la forêt (s.e. sacrée) » d'où ils proviennent, ils sont donc sacrés. Leurs avatars semi sacrés, les masques laids («masques maladies» dits encore bouffons ou comédiens) sortent du même lieu tandis que les masques d'amusement, lou-yu, dits «masques de femmes ", viennent du village et sont profanes (Haxaire 2009). Chaque masque possède une fonction réparatrice spécifique dont on peut aborder l'analyse par les propriétés attribuées aux entités végétales ou animales qui lui sont associées par contiguïté ${ }^{10}$. En effet, si ces masques de la famille Zamble dits « sacrés » sont sortis du bois en plein jour et peuvent être vus des femmes, il n'est pas permis à ces dernières de percer directement le mystère de leurs accessoires. Néanmoins, et ce sera notre propos ici, le savoir encyclopédique donne un biais permettant d'accéder au sens qu'ils véhiculent, car le propre du secret est précisément de résoudre la tension qu'il recèle en laissant, entre autres, sourdre des " sécrétions ", bribes d'informations émises et observations aléatoires permises à l'intrus destinataire qu'est l'ethnologue (Zempleni 1996).

\section{Zamble : son « mythe » officiel, et les versions ésotériques}

3 Le « mythe » d'origine de Zamble a été rapporté successivement par plusieurs auteurs ${ }^{11}$. Un chasseur aurait rencontré et capturé un yo " génie », dont il ne savait pas lorsqu'il

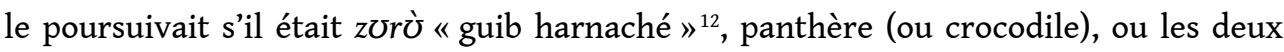
animaux mêlés lors de l'attaque. Il l'aurait caché dans le bois sacré en jurant de tuer toute femme qui $\mathrm{y}$ pénètrerait. Malheureusement ce fut sa propre mère qu'il dut exécuter. Son nom en est issu : zabll signifie celui qui a « mangé son maitre » au sens de celui qui a transgressé l'interdit de son culte. L'aspect du masque rappelle son histoire. Boti $^{13}$, le sculpteur de Tibeita, conçoit le masque comme une combinaison de l'antilope et du léopard ${ }^{14}$ car "Zamble est un léopard, la gueule ouverte juste derrière l'antilope qu'il poursuit ». On le dit courir aussi vite que le léopard tout en gardant la grâce de l'antilope. C'est pourquoi « le danseur de Zamble a, dit-on, l'intelligence de l'homme, la vélocité de la panthère et l'élégance de l'antilope ». Rapide, légère et gracieuse, cette danse doit s'accorder exactement à l'orchestre; c'est à l'exécution de ses pas que l'on juge un danseur.

4 Deluz (1992) nous rapporte des versions plus ésotériques de deux grands chanteurs, Bolia et Mazu-bi-Tra de la région nord ${ }^{15}$ du pays Gouro. Tous deux voient l'origine de Zamble dans un "petit » culte rapporté de chez les Nyãn au début du XvIII ${ }^{e}$ s. par un dénommé KasorJ ${ }^{16}$, enfant de Sei (au nord-ouest du pays Gouro). Ce dernier, récemment installé dans la tribu, était vexé de se voir exclu du partage des repas sacrificiels des autres membres de son village d'accueil, alors que de son côté, il les invitait; devant ce signe évident que son culte était méprisé, il retourna chez ses

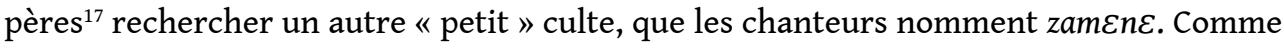
dans les versions précédentes, la propre mère de Kasoro pénétra dans la case du

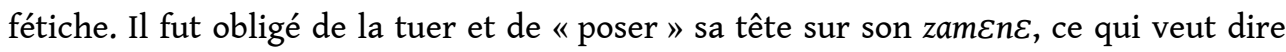


qu'il l'a offerte au fétiche, ce sacrifice contre-nature donnant ses assises à la puissance du culte.

5 Pour le sociologue gouro Zamble bi Zou (2009), ce fétiche était un za ma le « ce qui permet d'être puissant dans les confrontations", dans les guerres (guli) ou leurs substituts, les joutes inter (ou intra) villageoises. En effet, dans cette société segmentaire, acéphale, les guerres étaient nombreuses entre lignages ${ }^{18}$, pour un territoire, pour une femme, pour une insulte reçue. Mais elles ne concernaient que de petits groupes menés par leurs chefs de guerre qui devaient se "préparer ", ainsi que leurs troupes, c'est-à-dire se munir d'objets puissants (les fétiches), faire des sacrifices pour s'assurer la protection des ancêtres, se soumettre à des lustrations de plantes protectrices, ou ingérer des remèdes leur assurant force, endurance et courage. Depuis la pacification du pays sous le joug du colonisateur ${ }^{19}$, les défis, les compétitions dites " concurrences", se sont déplacées dans le champ des concours de danses de masques; les plus risqués mettent en lice des masques Zamble, dont les porteurs, tout comme les guerriers d'autrefois, doivent renforcer leur puissance et se doter de protections (Bouttiaux 2004). C'est à l'occasion des grandes cérémonies de levée de deuil que les familles les plus riches offrent ces performances à l'assistance accourue nombreuse ; mais il faut bien entendu des joutes intra-villageoises préalables pour sélectionner les meilleurs danseurs susceptibles de représenter le village ou la tribu «à l'étranger ».

Selon Zamble-bi-Zou, Kasoro, encore appelé Sein $\varepsilon$ ou Sei br, était un neveu utérin $(y \mho r \mho)^{20}$ de Banti-bi-Blon (Bati-bı-Blō) de la tribu Nyan. Ce grand guerrier, lui-même descendant de Gohi-bi-ta, le fondateur de la ville de Gohitafla, n'avait de cesse de provoquer des conflits pour conquérir de nouveaux territoires. KasorJ ayant tué la sœur de Banti-bi-Blon, ce dernier en représailles lui arracha ce fétiche contre toutes les règles gérant les rapports oncles utérins-neveux, parce qu'il le trouvait fort utile pour ses conquêtes. C'était un puissant fétiche protecteur qu'il sortait parfois à travers le village, couvert de jeunes feuilles de palme, dans le seul but de purifier, de protéger la communauté des forces maléfiques. L'auteur poursuit: ce n'est que lorsqu'ils eurent vaincu et assuré leur domination sur les autres lignages, qu'en guise de reconnaissance, les anciens, descendants du lignage de Banti-bi-Blon, décidèrent d'en faire un masque et de le faire danser.

\section{Zamble : ses surnoms, ses attributs}

7 Zamble est surnommé bila goli, «le léopard au beau pagne traditionnel rayé ». Goli, traduit ici par léopard ${ }^{21}$ est, selon le conteur Michel Zou ${ }^{22}$, un terme générique désignant tout animal qui mange les autres animaux, un « dévoreur ». Le crocodile est ainsi le goli des eaux (yı $\varepsilon i ́$ goli) ${ }^{23}$, plé l'épervier ${ }^{24}$ le goli des airs ${ }^{25}$. Mais cette catégorie exclut les petits. Parmi les carnivores $\mathrm{Gu}$, la nandinie ne serait pas un goli, mais un $k w \varepsilon n \varepsilon$ (petits mammifères carnassiers en général). Le lion ${ }^{26}$ jela, l'hyène ${ }^{27}$ glàú sont, avec la panthère kua, les goli du domaine terrestre en quelque sorte, mais les chants peuvent les mettre en correspondance avec leurs homologues du monde des eaux ou de celui des airs. Les plus puissants sont encore désignés en gouro par zowùli ${ }^{28}$. 
Figure 1 : Zamble lors de funérailles à Bogopinfla

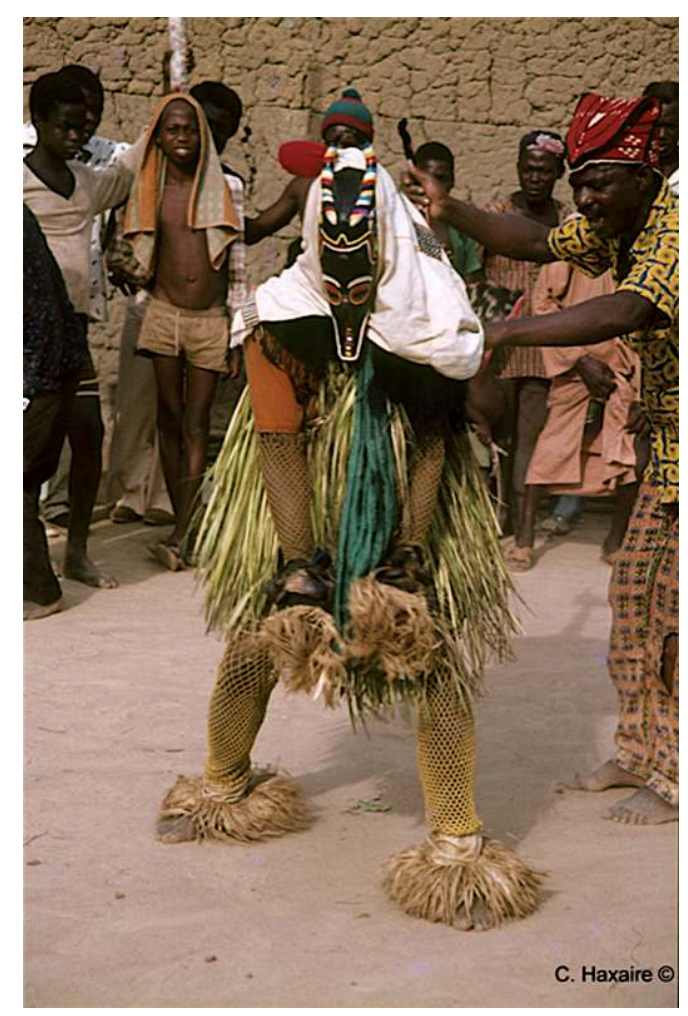

8 Les traits fins et les atours soignés de Zamble le présentent comme un être policé, le frère plus civil de Zàùlì, ou sa femme. Pour venir au village devant les femmes il s'est habillé d'un beau pagne. Les chants spécifiques du masque, par exemple «la panthère kua est assise sur une termitière ", appuient ce caractère. En effet, la panthère, qui apprécie la propreté, aime faire le guet sur les termitières, espaces en général dégagés. L'ensemble comporte un troisième masque, Gù. Lorsque les trois paraissent, Zàùlì est le frère sauvage (ou le patron) de Zamble accompagné de son épouse Gù. Cependant certains maintiennent que Zamble est la femme de Zàùlì tout en restant le mari de Gù, ce qui en fait donc un être de sexuation pour le moins ambivalente. Le masque représente un être composite, cornes d'antilope et gueule ouverte de léopard (goli); il porte d'ailleurs la peau d'un carnivore. Son costume de jeunes palmes sur le haut du corps en fait un être de la brousse. Sa courte jupe de raphia, les filets qui lui couvrent les jambes, les sonnailles qui ornent ses poignets et surtout le joli pagne entourant le masque, pendant sur la nuque et les épaules, signent son appartenance au village. Néanmoins Zamble n'est pas sans être accompagné du porteur du fouet.

Certains des attributs ou des chants de Zamble en font un culte de fécondité. Zamble porte dans le dos une petite bourse rouge en forme de fruit de Zabll-wuo-ma-klo «le petit chapeau de Zamble», un Cnestis ${ }^{29}$. Selon les Gouro, les feuilles de cette plante buissonnante sont emménagogues, c'est une des plantes «donnant de la force aux femmes » (ll-do-plele) c'est-à-dire augmentant leur sang. Elle entre dans la catégorie des bòj̀-le-goi « kola du gros rat-palmiste ${ }^{30}$ ", la kola donnant de la force à cet écureuil. Pour les femmes, cette fécondité se décline sur le mode de l'abondance du sang et de la jouvence, de la même façon que devient féconde une terre arrosée de pluie. Les chants de Zamble qui assimilent serpents et masques font référence au serpent le plus gros, qui «se bat avec l'homme », le boa mlenE sa «le serpent lumière » réputé se cacher 
pour manger ses proies dans les mares. L'arc-en-ciel bò̀̀-ml $\varepsilon$ qui surgit après la pluie et passe "rouge et noir» dans le ciel, est l'émanation de la puissance (fulu) du génie serpent, lové sept fois, et qui habite dans une grosse termitière, point d'entrée vers le monde souterrain des ancêtres. Son nom en ferait le serpent du même rat-palmiste bj̀̀, selon J.-P. Benoist (1978), cet écureuil empruntant peut-être l'arc en ciel comme chemin vers le ciel au même titre que le palmier. L'arc-en-ciel aux multiples couleurs est, dans les chants, associé à Zamble couvert de pagnes colorés.

Mais ce miroir chatoyant ne devrait pas nous masquer les aspects moins paisibles de ce personnage, ce que rappellent d'autres chants. Zamble est bien entendu un chasseur "Goli est un animal qui attrape le guib harnaché, qui ne peut le voir sans le tuer ». Mais c'est surtout un guerrier "goli est un nana za guerrier, c'est guli la guerre qu'il aime ", et le chant "la panthère est assise sur une termitière" se poursuit en fait par "qui peut l'approcher?»; c'est un chant de "concurrence » que l'on entonne lors des danses quand il apparait que le danseur de Zamble, que l'on soutient, domine nettement l'adversaire. Il renvoie au fait que les grands guerriers, puissants grâce aux "médicaments» qui les préparent au combat, ne pouvaient être côtoyés par des individus ordinaires plus faibles qui risquaient de voir leur jale "force vitale" annihilée par celle des guerriers. Il nous est bien précisé que tous ces masques étaient des armes (des choses) de guerre autrefois. On faisait la danse Zamble avant d'aller à la guerre. Il en découle que, pour les compétitions/concours de danse actuels, tout le monde se "prépare » de même. Il s'agit, avant la danse, d'une part, de se protéger soimême ainsi que le danseur ce à quoi les « féticheurs » de la troupe sont attentifs tout au long de la prestation, et de l'autre à préparer des «médicaments» (s.e. des poisons, Haxaire, sous presse) contre l'adversaire. Un chant en apparence aussi anodin que «le soleil décline à l'horizon" s'entend comme un signal codé : c'est le moment de lancer yひ dư « le poison de contact » contre l'adversaire, car il sera trop tard pour que son féticheur puisse aller en brousse chercher le remède, l'obscurité l'en empêchera.

11 En vérité Zamble est méchant "Goli est méchant, il aime la guerre » proclame le chant. Et son « frère sauvage » Zàùli, l'est-il davantage?

\section{Zàùli : son origine, ses attributs}

Zàùli, encore surnommé Gwa ou Gogo Yiere ${ }^{31}$, est bien donné comme beaucoup plus ancien que Zamble. Pour Sabu-bi-Seyi (cité par Fischer \& Homberger 1985, repris dans Fischer 2008)

«Il fut le premier à danser au village, bien avant les deux autres qui sont venus plus tard. Zàùlì est nettement plus puissant que Zamble, il vient pour mettre au net des choses assez difficiles, quand Zamble n'a rien pu faire et a été inutile ",

bref, lorsqu'il a échoué. En 1960 déjà, on avait dit à Himmelheber que «Zàùlì était si sauvage qu'on ne le laissait plus venir dans le village, qu'on ne l'avait plus vu depuis assez longtemps » (Himmelheber 1966). Néanmoins dans les années 1980-90, qui ont vu le déclin de Gù, le masque a effectué plusieurs sorties auxquelles nous avons pu assister dans le village de Sabu-bi Sei, chez les Wagye. 
Figure 2 : Zàùlì, masque puissant parcourant le village de Bogopinfla en prévention du malheur annoncé

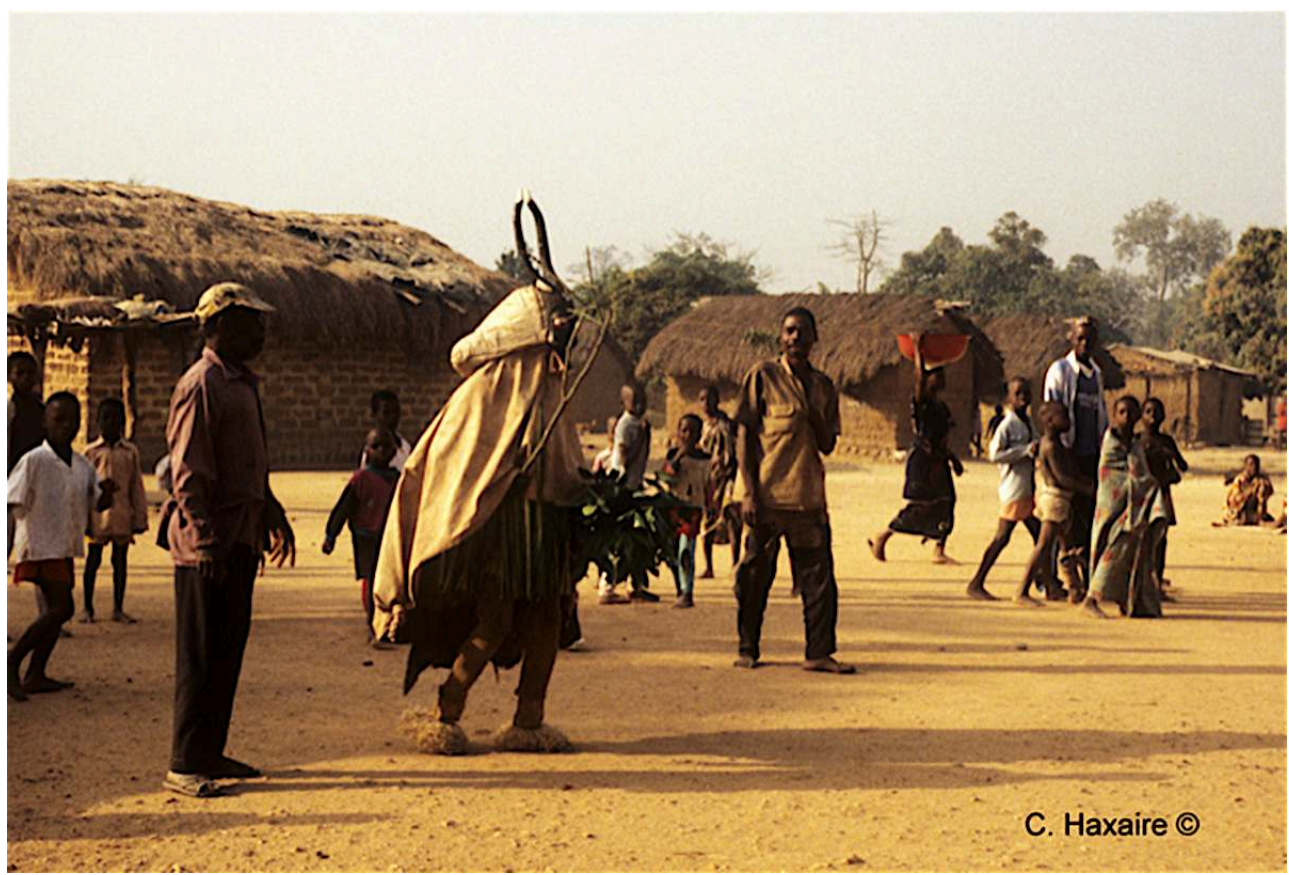

En pays Wagye et Yaswa, à côté des grands cultes comme $j \varepsilon, v l o, j i$ qui interviennent spécifiquement pour des évènements concernant les familles détentrices de ces cultes, il semble que, pour des malheurs collectifs, un masque doive sortir du bois sacré et apparaître en public. Du moins est-ce ainsi que Zàùlì nous fut présenté par Boti : «jE sors sous la stricte responsabilité des hommes; Zàùlì, que les femmes voient, protège celles-ci des dangers tout comme il protège les adultes et les enfants. C'est pourquoi lorsqu'il y a des problèmes, c'est lui que l'on fait sortir, et tout le monde le voit pour qu'il les protège tous ». Le danger collectif peut être une épidémie (autrefois de variole) ou, a minima, des vols. Mais un danger plus vague, le rêve d'un villageois pressentant des actes de sorcellerie, voire un décès, ou leur simple annonce par un devin, demandent à être conjurés par toute la communauté. Zàùlì se met alors en tête du cortège des villageois, chacun portant un tison (le danger, la maladie) pour le jeter en tas hors du territoire et l'étouffer de boue (l'enterrer), maudissant le sorcier ${ }^{32}$ à l'origine du fléau. Chacun, à l'appel de Zàùlì, parcourt le village, muni de branchages pour exorciser le malheur; ils représentent les plantes médicinales, remèdes des maladies annoncées. Fischer et Homberger (1985) précisent que «le masque caresse avec ce bouquet les habitants du village qui l'ont gratifié d'un cadeau, sur le front et la nuque ; il leur promet protection contre les maladies ». Le cadeau le plus apprécié du masque serait les noix de kola dont il est si friand qu'il en croque en permanence, comme le trahissent ses joues gonflées qui lui ont valu le surnom de ko-bli-lu, « fille du rat-toto ». Selon cette étymologie populaire donnant à bli le sens de " gonflé », il aurait comme Kobli, le rat-toto, les joues gonflées des graines qu'il garde en réserve. Les enfants qui agacent le masque pour qu'il les poursuive avec la verge qu'il tient de l'autre main, se moquent de cette avidité : « koblilu, koblilu, donne-moi la kola, il a de la kola dans la joue ». Mais il ne s'agit pas seulement d'une gourmandise, la kola est, avec le vin de palme, l'offrande requise pour les sacrifices, que le masque agrée s'il la consomme (Haxaire 1996). De plus, cet aliment est explicitement un stimulant qui donne de la force, celle dont Zàùlì a besoin pour jouer son rôle de restaurateur de l'ordre des 
choses, (ce que les attributs du beau masque Zamble, comme la kola du rat palmiste, déclinent en mineur). Cette force est bien la force du sang, si l'on interprète les chants spécifiques de cette danse; tel celui qui doit être entonné lorsqu'on arrive chez l'organisateur de la danse : "Oh bei, bei lu bei, je suis arrivé à destination ». Bei ${ }^{33}$ est, pour les Gouro, un arbuste dont les branches feuillées ont la propriété d'augmenter le sang et, de ce fait, de faire grossir les bébés tout comme de faire venir les règles des femmes. Les chants spécifiques de Zàùlì reprennent les thèmes de l'eau déjà vus avec Zamble sur un mode plus dramatique : " on le casse [le canari], on le casse et on joue avec les morceaux giliyoyo, la grande tornade se prépare ». En dépit ou à cause de cela, Zàùli se montre plus puissant protecteur, comme le dévoilent les tiges épineuses de $z a-g$ ग d'Asparagus ${ }^{34}$, enroulées aux cornes du masque. Cette plante peut être désignée par le générique de son usage, (gonE-do-plele « ce qui donne de la force aux hommes ». Zàùli est fort, c'est le sens des surnoms Gwa et Gogo Yiere, que les enfants chantent pour le faire se rengorger sous les louanges. Cette puissance se comprend comme étant celle de la force vitale jale, émanation de celle du sang, car za-go est également l'un des remèdes donnés aux concurrents de joutes ( $z a k l \varepsilon$ ), danseurs, chanteurs, conteurs, orateurs, qui doivent résister au jale du public, c'est-à-dire à la puissance qui se dégage du cumul des puissances dont sont porteurs les spectateurs. Sa tige mâchée en cure-dent par les danseurs en est le «contre-poison». L'Asparagus arboré par le masque se nomme en gouro $z a-g$ ? "plante des discussions", selon le vieux sage Fua-bi-sei. $Z a$ peut être traduit par "concurrence ", ou "pari», ce qui donne lieu à de multiples jeux de mots dans les chants de Zàùli, par exemple : «Toi qui veux parier, voici un pari ». Pour le vieux sage cité, c'est par son ambiguïté que cette plante suscite les discussions : "C'est une liane, mais on n'attache rien avec, c'est une plante feuillée et elle a de toutes petites feuilles, n'est-ce pas un sujet de discussions? ». Mais puissance et ambiguïté sont liées car cet Asparagus, tout hors norme qu'il soit, ou peut-être précisément parce qu'il ne répond pas aux critères de sa classe, n'en détient pas moins une force remarquable puisque sa base souterraine peut être utilisée comme support pour couper les boutures d'ignames (des meilleures variétés). L'Asparagus za-go्N, bien en évidence à la pointe des cornes du masque, affiche la nature sauvage de cette entité, jouant résolument de la confusion des genres, du désordre dont il tire sa puissance. Ainsi, dit-on, "Zàùlì est puissant pour pouvoir protéger les gens ». Il a le pouvoir d'éliminer par sa malédiction le responsable du malheur, mais peut intervenir pour restaurer l'ordre car ce sont les disputes, les conflits, qui déchirent la cohésion sociale, et laissent place à l'action des sorciers.

La venue du masque suscite des comportements peu civils. Aujourd'hui, les membres de l'assistance peuvent se barbouiller mutuellement de charbon. Autrefois c'était du piment mélangé ou non à de la kola qu'il leur arrivait de se cracher dessus, tout comme lors des grands rituels, les $y \sigma-k l \varepsilon$-za féticheurs projetaient ainsi des remèdes « contrepoison » sur l'assistance. Comme tout génie de brousse, être de désordre, Zàùli serait si laid qu'il s'en ferait cracher dessus par les sacrificateurs. Mais ne nous y trompons pas, cet acte confère au masque la puissance de leur souffle, support de jale, tout comme, en retour, les accolades dont Zàùlì les gratifie augmentent la force vitale de ses vieux adorateurs.

16 La laideur est donc un attribut du masque, inhérent à sa fonction. Son front, loin d'être lisse et bombé selon les canons esthétiques gouro, serait fendu (vlغ્ָi) à l'égal de kású, le bubale $^{35}$ dont les cornes écartées déforment la face. Le masque de notre village de référence, masque zoomorphe, porte les cornes du plus dangereux des cobes, glò le 
cobe defassa ${ }^{36}$, le plus puissant des animaux pour lesquels les chasseurs doivent mettre en place le rituel de purification Jale. De plus, Zàùlì a de gros yeux globuleux, une peau couverte d'excroissances, séquelles de maladie. Laid, il ne peut que porter de vieilles hardes, des so wuli pagnes usagés, il s'est fait wuli « chose vieille » d'où son nom selon une autre étymologie populaire (zàà-wuli ou encore $y \sigma$ wuli, « vieux masque abîmé »). Il porte sur la nuque, au lieu du petit bonnet rouge qu'affectionnaient les anciens, un gros sac en forme de vieux couvre-chef démodé et élimé. Comme tout masque sacré, il est vêtu de fibres fraîches de jeunes feuilles de palmier, mais son costume lui laisse les mollets à découvert. Dans le dos, ce vieux sac couvre une solide ${ }^{37}$ peau de gà̀, le cobe de Buffon, que soutient son accompagnateur. Il convient de lui donner des choses dures $(p l \varepsilon l \varepsilon)$. Parce qu'il se bat, fait la guerre, rôle décrit plus haut pour Zamble. Un servant de sa troupe manie un long fouet qu'il fait claquer pour chasser les enfants, mais aussi éloigner les êtres malfaisants.

Son comportement est à l'avenant, désordonné, poursuivant femmes et enfants de sa verge fourchue. Il apparaît sauvage, ne respectant pas les usages. Zàùlì, qui pourrait danser comme Zamble, se contente souvent de pas lourds, peu rapides, plus mime grotesque que danse, reflet du personnage. Il passe de cour en cour et répond aux déclarations du maître de maison en frappant le sol du bouquet de feuilles et de la verge qu'il tient en main, agitant les sonnailles de ses poignets.

\section{Son surnom « fille du rat-toto », renvoie à l'histoire secrète de Zamble}

Zàùlì, surnommé kobli-lu, fille du rat-toto, habite la forêt sacrée, la brousse, mais fréquente le village tout comme son parent kobli, le rat-toto. Ce dernier a la réputation de ne sortir qu'à l'occasion de problèmes et dans ce cas n'a de cesse de rendre visite à sa parente, flaù, la petite souris de case ${ }^{38} \mathrm{si}$ au fait des secrets de famille qu'un type de divination l'utilise comme médium. Zàùlì assure le lien entre brousse et village. Un chant spécifique marque cette situation incongrue pour un masque sacré de se présenter devant les femmes "C'est une femme qui t'a mis au monde, ne te frappe pas la poitrine [ne te fâche pas] à cause d'elle ». Mais il faut bien qu'il soit fille (lu) pour justifier cette apparition publique d'un masque de la famille $j \varepsilon$, du moins dans les régions étudiées. Selon Fischer et Homberger (1985) "pour de nombreux Gouro, Zàùlì appartient au même groupe que le masque que ces auteurs nomment 'gye' (jع) ». Ce que recoupent également nos informations, obtenues certes très furtivement. C'est d'ailleurs ce que laisserait entendre son surnom, si le rat-toto est bien vlù br koblì «fils du porc-épic», et si nous suivons Boti, pour qui $j \varepsilon$ serait un masque (ou les $y \delta$ ) d'antilope et de porc-épic. Zàùlì serait donc "semblable à 'gye' " (je) et aurait les "mêmes traits de caractère » masculin, sauvage, « devant inspirer la peur et pour cela ne pas être vu des femmes ». Boti précise qu'ils ont plusieurs couleurs, les mêmes yeux et un nez comportant une arête ${ }^{39}$. Cette figure serait «sortie du bois sacré » pour se laisser voir par l'ensemble du public dont les femmes.

Prenons alors au sérieux le surnom de Zàùlì et voyons ce qu'il en est du rat-toto dont Zàùli est la fille, et qui est le fils du porc-épic (vùlu). On appelle également kobli, le rattoto, vulú br c $\varepsilon j \mathcal{E} \varepsilon$ " celui qui court vite et lourdement, fils du porc-épic », du fait de sa course certes très rapide mais sans élégance. Autrefois, nous conte Michel Zou, le rattoto ne s'appelait pas Kobli, mais plutôt Yowuliyo. C'est un animal qui passe partout, 
des champs au village jusque dans les maisons, puisant dans les réserves de graines, un fieffé voleur selon notre ami chasseur. Il reste à proximité des hommes et se repaît des graines de palmiste abandonnées dans la cour des campements, déchets de la préparation des repas. Il fait d'une termitière ${ }^{40}$ son terrier, pas très loin des villages ce qui permet aisément de l'attraper lorsqu'un étranger arrive et que l'on n'a pas de viande à lui offrir. Il suffit de boucher tous les trous de son terrier sauf un. C'est pourquoi on le désignait aussi comme "l'animal à longue queue que les étrangers aiment ». Dans le conte, sa termitière se trouvait à mi-chemin entre deux villages et dès l'aube, on l'entendait pleurer debout sur ce promontoire : « oh le village a commencé la guerre, village de mes parents, la guerre va te tuer» "oh il va me tuer », comme s'il était un homme posté au milieu des deux villages et qui criait pour avertir ses compagnons du danger. Chaque village pensait que c'était l'un des leurs qui pleurait ( wu vว $n \alpha$ ), les gens accouraient et cela devenait un affrontement, une bastonnade. Mais Yowuliyo était déjà rentré dans son trou. De guerre lasse, un des grands chasseurs (ceux qui marchent dans les herbes) est venu se poster très tôt le matin pour voir de quoi il retournait, et quand l'animal commença à pleurer, le grand chasseur a bouché son terrier pour qu'il ne puisse plus y rentrer. Il l'a ainsi attrapé, a fait un feu pour le faire braiser et l'a mangé avec ses premiers compagnons accourus. Quand les autres sont arrivés en disant " où est passé celui qui criait? », ils ont répondu « nous l'avons mangé » kứ bll . C'est pourquoi on chante « kobli, viens, on va s'amuser »; sinon avant il s'appelait Yowuliyo ${ }^{41}$. Kobli, le rat-toto, est donc un faiseur de guerre, tout comme autrefois nombre des ancêtres gouro cités dans les généalogies, tout comme Banti-biBlon.

Si le rat-toto est le fils du porc-épic, selon les chasseurs, c'est parce que toute cette famille, incluant télí l'athérure ${ }^{42}$, également vùlu-br-téli « fils du porc-épic », mange des choses amères, dont ziri, le poison d'épreuve ${ }^{43}$, qui tuerait les humains et bien d'autres animaux. Ils mangent également la plante vùlu le ya $a^{44}$, qui devient leur igname. Tous ne font pas de terriers. Le plus puissant d'entre eux, vùlu, le porc-épic, se gonfle (bli) et lance ses piquants pour les Gouro. C'est du gros porc-épic que l'on a peur. C'est le devin des animaux, mais pour aller le consulter lorsque l'on ne parle pas sa langue, il faut passer par un enfant de sa sœur ou de son petit frère, comme porte-parole intermédiaire, c'est-à-dire par un neveu. Et c'est $c \mathcal{c} n \varepsilon$, Kobli qui tient ce rôle. Le conteur Bueti-bi-Dje ${ }^{45}$, précise bien que Kobli est un fils classificatoire du porc-épic, en réalité un neveu, l'enfant de sa sœur, ce qui explique que le rat-toto soit plus petit que le porcépic et qu'il n'ait pas de piquants ${ }^{46}$, tout comme l'athérure a d'ailleurs des piquants différents étant le fils d'un petit frère. On voit ici intervenir Kobli comme neveu du grand guerrier qu'est Vùlu, tout comme Kasoro l'était de Banti-bi-Blon.

21 Zàùli fille du rat-toto, lui-même neveu du grand guerrier porc-épic, transpose donc l'histoire originelle de Banti-bi-Blon à qui sait entendre, à qui détient le savoir permettant d'en décoder le sens, voire d'en jouer. Zàùli assumerait ainsi le côté guerrier de Zamble, ce qui aurait permis au masque éponyme de se présenter sous un jour raffiné, voire féminin.

\section{Et la femme gù ?}

À Zamble succède parfois la danse de Gù au seul rythme des clochettes de ses pieds et des cris d'encouragement du public. Banti-bi-Blon, marié avec une femme wan, une 
ethnie voisine au nord, l'aurait adopté de ses beaux-parents ${ }^{47}$. Gù, au visage de femme lisse, toujours noir, au front bombé, est souvent coiffée de sept petites cornes qui interdisent de porter sur la tête, indiquant son statut social. Vêtue comme son mari Zamble d'une courte robe de fibres croisée sur la poitrine, elle couvre ces attributs de la brousse de pagnes bleus richement tissés. Étant elle-même précieuse (nunimi-a), Gù ne porte que de belles choses. Pour Benoit (1978), elle serait surnommée célizia-lú-mòo, mòว: une fille très belle, au teint clair, donnée en mariage à un esclave sans compensation matrimoniale, ce que faisaient autrefois certains pères pour garder la descendance et la fille dans leur lignage. Elle porte quant à elle une peau de cobe defassa ou de guib harnaché, peaux suffisamment solides pour que son accompagnateur puisse les faire gonfler $(b l i)$, les tendre avant de frapper dessus pour arrêter un cycle de pas, rappel de son origine wan car il en va de même pour la danse Goli des Wan.

Gú ne sort que pour les rituels de fécondité : installer le foyer d'une nouvelle épousée de sa famille, résoudre son problème de mal d'enfant et, selon Deluz (1993), «la protéger de toute malveillance de la part de la famille où elle est mariée ». Les cinq villages Wagye partagent un ensemble de sept masques spécifiques, yoro, nommé « gù des waje ", donné comme étant sorti d'une grotte à flanc de colline et capturé par un chasseur. On doit leur donner un poulet au moment des funérailles d'une fille de la famille. L'un de ces masques tient en main la plante galactagogue, $m \mathcal{E}-$-pó « genoux de poulet $»^{48}$ surnommée gù-lèè-bla, "la plante que gù tient en main", qui soigne également les suites d'accouchement. Mais yoro peut alternativement, ou de façon associée, tenir bowolo ${ }^{49}$, plante utilisée pour soigner des atteintes de l'ombre-double du nouveau-né ou de quiconque a offensé les ancêtres. Ce qui décline, sur un mode mineur, les propriétés de l'Asparagus, apanage de Zàùlì, car il s'agit dans tous les cas de protéger ou de renforcer l'ombre-double.

Lorsque Gù apparaît lors de funérailles, elle suit toujours Zamble; les tambours se taisent et elle danse très gracieusement au son des seules flûtes. Sa danse est dro-dro calme, littéralement fraîche, ce qui cadre avec l'apaisement et la jouvence que sa venue apporte. Elle marche majestueusement telles les belles femmes. Pour A.M. Bouttiaux (2005) elle piétine comme une enfant gâtée les graines de palmistes. Pour montrer sa force nous dit-on, peut-être aussi piétine-t-elle ce qui symbolise sa dot dans les contes, soit les graines du palmier (Haxaire 1992), elle que son père aurait dédouanée du poids de cette dette. Un chant caractérise ses pas de danse «dúú, le francolin ${ }^{50}$, s'envole brusquement, avec force (za), mais se pose doucement à son point d'arrivée glìglì ». C'est un chant de concurrence (bien que Gù ne participe à aucune compétition) : s'élancer trop vite en étant trop sûr de soi risque d'amener des déceptions si l'adversaire est plus fort que prévu. D'autres chants évoquent le travail domestique de la femme, rythmé par le chant du coq. Elle se lève au premier chant du coq, balaye la cour, va puiser de l'eau... "mon frère ne manque jamais to le temps ... la tête d'un coq dans la sauce... ". Selon le chanteur Irie-bi-Dje Medah, le coq a le temps en tête, on peut se fier à lui. En manger la tête permettrait de se réveiller à l'aube. Les chants conseillent de se résigner aux mariages forcés, qui peuvent s'avérer heureux avec le temps «ban£ lu yoñ $\alpha$, la grande menteuse, yonan est venue ». Younan aurait refusé le mari qu'on lui promettait avec la dernière énergie, elle a fui. Mais ses parents l'ont rattrapée et à force de " médicaments ", de boli, les charmes d'amour, et en faisant appel à Zamble, Zàùli et Gù pour installer son foyer, elle est restée. Elle a eu des enfants, des petits-enfants et toute sa famille est accueillie dans sa cour prospère et heureuse. La vie l'a fait mentir ! 

peut-être n'est-elle qu'un avatar de la nandinie gu (ton moyen) elle-même surnommée, selon Benoist (1978), zablı tí br gu "nandinie, fils du père de Zablı ». Le terme Zablı n'évoque rien aux Gouro de Zuénoula, mais on le retrouve dans le nom composé de l'aigle huppard tel que recueilli dans la région de Sinfra par l'ethno-ornithologue gouro Mathieu Koué bi (2016) : cle le zable, le zable de l'aigle ${ }^{51}$. Cet auteur a également colligé les noms d'oiseaux dans la région de Zuénoula, et ce même aigle huppard devient zowùlì bl $c l \varepsilon$, soit un des aigles-fils du plus puissant parmi les oiseaux qui tuent les autres. Si nous transposons dans le domaine des carnivores, ce serait bien la nandinie, fils $\mathrm{du}$ père du plus puissant des goli, du zowùlì des goli, à savoir Zàùli, issu donc de $j \mathcal{k}$. Gu serait donc fils (fille) de $j \varepsilon$. Le cri de cet aigle huppard est un mauvais présage. Pour le conteur, Gu la nandinie, plus que le fils de cet aigle, serait son homonyme, partageant

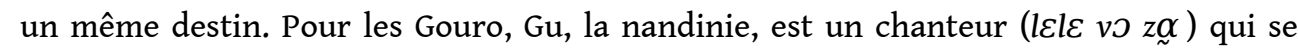
cache quand il fait jour et vit dans la forêt, en lisière ou dans les îlots de forêt en savane. Quand la nuit tombe il grimpe aux arbres et de leur cime crie, crie, en faisant comme quelqu'un qui pleure wu wu... Les contes glosent cette tendance à « pleurer » de la Nandinie. Elle aurait convenu avec vla, «le daman $»^{52}$, de s'interdire de pleurer lors des funérailles de leur mère. Ce qu'est parvenu à faire le daman. Mais ce dont la nandinie a été incapable, elle n'a pu tenir sa promesse et s'est cachée dans les arbres pour gémir doucement wu wu. C'est alors que le daman lui a crié (gu é wúvoa) «Gu pleure? ", et c'est devenu le chant du daman.

Gù certes femme, qui devrait se soumettre aux règles qu'énoncent les chants, n'en est pas moins affranchie par son père, et tout comme son mari et/ou père, cache sous sa belle apparence la violence de ses caprices de $y \sigma$. Fille du plus puissant des masques tel que le révélerait le nom de l'animal homonyme de l'aigle, elle sait d'autant mieux trouver recours aux malheurs de femme qu'elle en connait les présages.

\section{Conclusion}

27 Comme toujours en pays Gouro, les chants et les contes ont une signification cachée, qui joue des analogies que permettait la grande connaissance naturaliste qu'avaient les anciens. Les mascarades, en tant que productions artistiques totales, et cela d'autant plus qu'elles dévoilent quelques bribes du sacré, présentent actuellement le miroir de récits d'une tradition en train de se réinventer, laissant ce contenu manifeste repousser dans les brumes du passé l'histoire magnifique et violente des guerriers d'autrefois. Les surnoms de ces masques n'évoqueront bientôt plus grand chose, et les interprétations de leurs noms même iront bon train, permises par la spécificité de la langue gouro. Cette langue semble autoriser des dérives autour de contigüités cachées grâce à divers procédés de dissimilation qu'il faudrait étudier. Seuls les tons par exemple distinguent $z a$ « défi » de zá " colère ", dont nous sentons bien la proximité ; il en est de même de $z J 0$, «querelle » et ż̀̀, « sorcellerie ». Ce pourrait être le cas de gu la nandinie et du masque gù. Les conteurs vont plus loin encore qui font dériver le nom du rat-toto de l'expression « nous l'avons mangé » en fermant les voyelles relâchées. Tout ceci révèle la créativité non seulement de la langue mais surtout de la culture gouro.

Certes il n'est pas facile à qui n'est pas familier de cette culture et de cet environnement de suivre le dévoilement des significations de ces chants, de ces contes, des mascarades toutes entières. Mais j'ai plaisir à partager ce florilège d'histoires 
auxquelles manque cependant, et je le regrette, l'explosion de couleurs et de musiques des danses qui les mettent en scène.

Figure 3 : Zaouli, danse d'amusement lors de fête de levée de deuil en 2018 dans le village de Bogopinfla

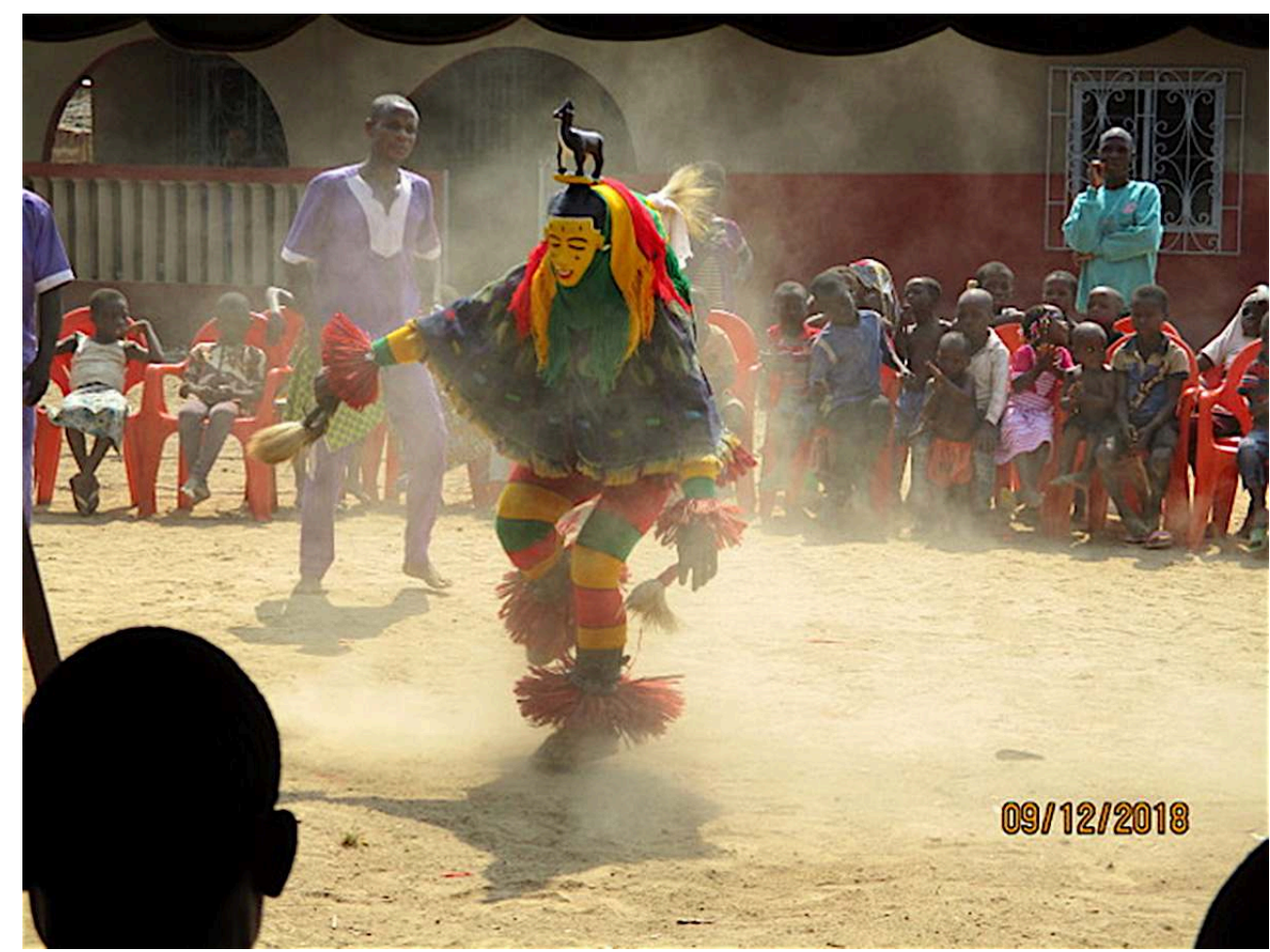

Ce travail fruit de dizaines d'années d'enquête doit à tous les Gouro qui m'ont instruite, et je salue la mémoire de ceux qui nous ont quittés. Je voudrais particulièrement remercier les conteurs Bueti-bi-Dje assisté de Paul Tra bi et Gala-bi-Yuzan alias Michel Zou ; le chasseur Goi bi Tra et les gardes forestiers Irie bi Sela Mathieu et Tra bi Mattias; le chanteur Irie bi Dje Medah toujours disponible pour nous donner des explications, et bien entendu Benin bi Dje, fidèle et rigoureux assistant décrypteur.

\section{BIBLIOGRAPHIE}

Augé M. 1986 - Anthropologie de la maladie. L'Homme 97-98 (26) : 81-90.

Benoist J.-P. 1978 - Dictionnaire Gouro Français. Mission Catholique de Zuénoula (Côte-d'Ivoire).

Boulore V. 1995 - Les masques Baoulé dans la Côte d'Ivoire Centrale : approche historique et stylistique. Ph D diss, Univ de Paris I/Pantheon Sorbonne.

Bouttiaux A.M. 2004 - Porteur de Zamble en pays Guro : les enjeux de la célébrité. In : Fergombi A. (Ed.) Les rites et les arts. Revue d'Arts plastiques et d'arts du spectacle, Valencienne : Université de Valencienne $1: 76-85$. 
Bouttiaux A.M. 2005 - Gu, belle et turbulente, Un masque des Guro de Côte d'Ivoire. Arts et Culture $6: 134-151$.

Deluz A. 1970 - Organisation sociale et tradition orale, les Guro de Côte d'Ivoire. Paris-La Haye, Mouton.

Deluz A. 1989 - Gouro et Yohouré. In : Corps sculptés, corps parés, corps masqués. Chefs d'œuvre de Co Ste d'Ivoire, Catalogue de l'exposition du Grand Palais, Paris, octobre à décembre:132-141.

Deluz A. 1992 - Le meurtre de la mère dans le culte du Zamble. Journal des Africanistes 62 (2) : 183-191.

Deluz A. 1993 - Les Gouro. In : Barbier-Mueller J.-P., Arts de la Côte-d'Ivoire dans les collections du Musée Barbier-Mueller. Genève, Musée Barbier-Mueller : 234-245.

Evans-Pritchard E. 1940 - The Nuer. A Description of the modes of livelihood and political institutions of a Nilotic people. Oxford, Clarendon Press.

Fischer E. 2008 - Guro. Zurich, Museum Reitberg \& Preste.

Fischer E. \& Homberger L. 1985 - Masken Gestalten des Guro, Elfenbeikunste. Zurich, Museum Rietberg.

нахаire C. 1992 - Le palmier à huile chez les Gouro de Côte-d'Ivoire. Journal des Africanistes 62-1 : 55-77.

нахаire C. 1996 - Le vin de palme et la kola, nourritures paradoxales, médiateurs de la communication avec les dieux. In : Hladik C.M., Pagezy H., Linares O.F., Hladik A., Koppert G.A. \& Froment A., L'alimentation en forêt tropicale : interactions bioculturelles et perspectives de développement. Vol II : Bases culturelles des choix alimentaires et stratégies de développement. Paris, UNESCO : 101-116.

Haxaire C. 1998 - 'L'arbre resterait-il vivant s'il ne respirait pas ?' Conception du vivant pour les Gouro de Côte d'Ivoire, exemple de l'arbre. Anthropologica 40 (1) : 83-98.

Haxaire C. 2009 - The Power of Ambiguity. The Nature and Efficacy of the Zamble Masks, revealed by disease masks, among the Guro people (Ivory Coast). Africa 79 (4) : 543-569.

Haxaire C. 2017 - Quand la semence se fait poison : organisation de la pharmacopée Gouro autour des troubles de la fécondité. In : Gutierrez Choquevilca A.L. (Ed.) Guérir, Tuer. Numéro spécial Cahiers d'anthropologie sociale $14: 87-111$.

Haxaire C. (sous presse) - Yu duun, fétiches lancés ou poisons de contact ? Vivre sous le regard des ancêtres et la menace du poison en pays Gouro (RCI). In : Cros M. \& Bondaz J. (Ed.), Pharmakon : Mondes empoisonnés, approche anthropologique.

Himmelheber H. 1960 - Negerkunst und Negerkünstler. Braunschweig, Klinckhardt und Biermann.

Himmelheber H. 1966 - Guro ‘dje’Maskentanz. In : Encyclopedia cinematographica. Göttingen, Institut für den wissenschaftlichen film.

Himmelheber H. 1993 - [mit Fischer, Eberhard und Barbara und Ulrike Himmelheber]. Boti, ein Maskenschnitzer der Guro, Elfenbeinküste. Notizen zu Persönlichkeit, Werkverfahren und Stil eines traditionellen Bildhauers in Westafrika. Zürich, Museum Rietberg.

Kacou V. 1978 - Les masques et leur fonction sociale chez les Gouro. Annales de l'université d'Abidjan, serie F (ethno-sociologie)/VII.

Koue Bi Tih M. 2016 - Aperçu sur l'avifaune de la région de la Marahoué et ethno-ornithologie en pays Gouro (Centre Ouest de la Côte d'Ivoire). Thèse écologie animale, ethno-ornithologie, Université Félix Houphouët-Boigny, Abidjan, Côte d'Ivoire. 
Meillassoux C. 1964 - Anthropologie économique des Guro de Côte d'Ivoire. Paris-La Haye, Mouton.

Ravenhill P.L. 1988 - An african triptich: on the interpretation of three parts and the whole. In : Object and intellect : Interpretation of meaning in African Art : Art Journal 47 (2) : 88-94.

Roskov Y., Ower G., Orrell T., Nicolson D., Bailly N., Kirk P.M., Bourgoin T., DeWalt R.E., Decock W., Van Nieukerken E., Zarucchi J. \& Penev L. (Ed.) 2019 - Species 2000 \& ITIS Catalogue of Life, 26th February 2019. Digital resource at www.catalogueoflife.org/col. Species 2000: Naturalis. Leiden, the Netherlands.

Seignobos C. 2018 - Les termitières, un univers de chasse (Nord Cameroun). Revue

d'ethnoécologie [En ligne], 14 | 2018, mis en ligne le 31 décembre 2018, consulté le 13 mars 2019. URL : http://journals.openedition.org/ethnoecologie/3593 ; DOI : 10.4000/ethnoecologie.3593

Tauxier L. 1924 - Nègres Gouro et Gagou. Paris, Geuthner.

Zamble Bi Z. 2009 - Persistance des traditions face à l'invasion de la modernité: Étude du cas des masques Gouro des tribus Mass et Gnan de la sous-préfecture de Gohitafla. Thèse de sociologie, option : socioanthropologie des religions, Université de Cocody, Abidjan (21 oct 2009).

Zempleni A. 1996 - Savoir taire. Du secret et de l'intrusion ethnologique dans la vie des autres. Gradhiva $20: 23-41$.

\section{NOTES}

1. Que je n'ose nommer ballade!

2. «Masque " désigne ici les personnages, leurs costumes, leurs accessoires et accompagnateurs, et non les seuls objets collectionnés que les Gouro désignent par bois ou tête.

3. kua, Panthère-Léopard, Panthera pardus. Identifications vérifiées par Roskov et al. (2019).

4. goi, Kola, Cola nitida (Sterculiaceae)

5. kobli, Rat-toto ou Rat de Gambie, Cricetomys gambianus.

6. vùlù, Porc-épic, Hystris africaeaustralis.

7. Au moins épouse classificatoire dans la terminologie de parenté omaha.

8. gu, Nandinie, Nandinia binotata.

9. Je traduirai par la suite jale par puissance, attribut de l'ombre double, lei, issue des instances invisibles de la personne, différent de la force plele, en lien avec la quantité de sang pour les Gouro.

10. Dans le système de pensée non dualiste Gouro, masques et préparations à base de plantes n'appartiennent pas à des registres fondamentalement séparés et le découpage empirique/ magico-religieux déjà critiqué par Augé (1986) n'a pas de sens au regard des analogies qu'il s'agit de dérouler et de croiser dans le visible et dans l'invisible (Haxaire 1998, 2017).

11. Himmelheber 1960, Kacou 1978, Fischer et Homberger 1985, Deluz 1989 et 1993, Bouttiaux 2004 et finalement Zamble bi Zou 2009.

12. Zひrò, Guib harnaché, Tragelaphus scriptus, crie comme un chien et porte malheur, ce qu'exprime le chant : "Qu'est-ce que j'ai fait au guib zurò ? Où que j'aille, il crie ».

13. Sculpteur avec lequel Fischer et Himmelheber ont beaucoup travaillé et à qui ils ont dédié un livre (1éd 1993, rééd. 2018 ) (Himmelheber 1993).

14. Panthère et Léopard sont les deux noms en français de Panthera pardus, il s'agit du même animal. Par convention je traduirai kua par panthère et lorsque golí est utilisé, j'utiliserai Léopard.

15. A. Deluz 1970, chap I, différencie les tribus du nord, de l'ouest et du sud du pays Gouro, sur la base de différences de terminologie de parenté (Omaha au nord et Hawaien au sud), et d'autres 
critères comme l'habitat, les productions agricoles, la forme de dialectisation de la langue, les cultes. Les Gouro étant d'organisation segmentaire (Evans-Pritchard 1940), nous employons le terme «tribu » au sens précis où Meillassoux (1964) et Deluz (1970) l'utilisaient, c'est-à-dire une unité territoriale en relation d'alliance et de guerre avec les unités alentour. Les tribus Nyan tout comme les Yaswa et Wagye cités plus bas sont des Gouro du nord selon cette classification.

16. Ce prénom n'étant pas à consonance gouro, il laisse entendre en effet que cet homme serait originaire d'autres populations Mandé du nord-ouest du pays Gouro. On peut alors supposer qu'il s'agit d'un neveu, fils de donneurs d'épouse des Nyan.

17. Ce qui signifie donc qu'il avait suivi sa mère retournée dans son lignage d'origine après avoir été mariée chez les Sei. Dans l'organisation patriliénaire gouro, il est donc fils ou enfant de Sei (sei bl, ou seinE).

18. Autrefois les «tribus» étaient plus que maintenant constituées d'un nombre limité de lignages (Deluz op. cit.)

19. En pays Gouro la conquête s'est déroulée de 1906 à 1914 (Meillassoux 1964)

20. De terminologie de parenté Omaha les membres des tribus du nord du pays gouro se sentent redevables aux lignages qui leur ont donné des épouses et les relèvent d'un rang dans la parenté. En effet, les alliances matrimoniales se nouent entre lignages non alliés auparavant, idéologiquement « ennemis ». Les neveux utérins ont le rôle d'intermédiaires dans les guerres : pour demander l'arrêt des combats, ce sont eux qui affrontent l'ennemi avec le rameau blanc de jeunes feuilles de palmier, ils risquent leur vie. Par ailleurs ce sont eux qui creusent les tombes, etc. Le lignage utérin devient pour eux le refuge en cas de danger ou de conflit, ils y sont de plus épargnés en cas de vendetta; dans les généalogies nombre d'ancêtres ont ainsi quitté leur tribu d'origine pour s'installer chez leurs oncles maternels.

21. Car le français n'a pas de terme générique recouvrant cette catégorie gouro ; inversement on ne trouve pas en gouro de catégorie générique « antilope ».

22. Gala bi Yuzan alias Michel Zou, fils de Zamble bi Gala qui nous avait instruite autrefois, assisté de Irie bi Sela Mathieu.

23. yı દí goli, Crocodile, Crocodylus niloticus. C'est pourquoi le vieux Fua-bi-Sei de Bogopinfla, que Fisher et Homberger nomment Sabu-bi-Seyi, a pu dire à ces derniers que Zamble était un masque de crocodile et de guib qui dansait en luttant entre le fleuve, domaine de l'un, et la terre, habitat de l'autre.

24. ple્ć, Épervier shikra, Accipiter erythropus. Ou Buse à queue rousse, Buteo auguralis.

25. Il existe même une guêpe (qui ressemble à la guêpe maçonne) qui tue ses congénères et porte le nom de nono won $\varepsilon$ je golí "goli qui tue les insectes". (Il existe bel et bien des mouches carnivores telles que Rhagio scolopaceus, mais nous ignorons s'il s'agit de celle-ci).

26. jEla, Lion, Panthera leo.

27. glớv, Hyène rayée, Hyaena hyaena.

28. Le lion dans le conte de Michel Zou par exemple, l'aigle huppard dans les surnoms de rapaces (cf. plus loin).

29. Zabli-wuo-ma-klo « le petit chapeau de Zamble», Cnestis ferruginea (Connaraceae).

30. bj̀̀̀, gros rat palmiste, Grand écureuil de Stanger, Protoxerus stangeri.

31. Selon Zamble-bi-Zou il aurait été également adopté des Wan. Il reconstituerait alors un tryptique sur le modèle du Goli en vogue chez les Wan, décrit par Ravenhill (1988) mais Zamblebi-Zou réfute l'idée de tryptique car il introduit un quatrième culte, celui des yひnc. Nous ne retrouvons pas ceci chez les gouro de Zuenoula. Les emprunts de cultes et de masques étaient très courants dans cette région selon les historiens d'art (Bouloré 1995, Bouttiaux 2004), notre propos n'est pas de reconstituer cette histoire.

32. Au sens de witch.

33. Bei, Piliostigma thonningii (Caesalpiniaceae).

34. za-g2, Asparagus sp. (Liliaceae). 
35. kásó, Bubale, Alcephalus buselaphus.

36. gl’̀, Cobe defassa, Kobus (Kobus) ellipsiprymnus.

37. Tout comme on le fait pour Goli, masque d'une autre série, celle des wan, qui fait claquer son fouet sur cette peau. gá, Cobe de Buffon, Kobus cob leucotis.

38. Flaù, souris de case, Mus domesticus.

39. Le nom de ce culte signifie maladie, mort. Au sud du pays gouro, je est représenté par plusieurs masques. Le nom de l'un d'eux, Zahule, vieillard sale et négligé, couvert de coquilles et de plumes, au visage blanc d'où pendent des excroissances, n'est pas sans évoquer Zàùlì.

40. Bien décrit au Cameroun par Seignobos (2018).

41. Dans kúo bll, nous avons des voyelles relâchées, entre u et o, et entre i et e, notons que dans kobli les voyelles sont toutes refermées, o et i.

42. télí, Athérure, Atherrurus africanus.

43. ziri, le poison d'épreuve ou Bois rouge, Erythrophleum guineense (Fabaceae).

44. vùlu le yaa, « igname du porc-épic », Anchomanes difformis (Araceae).

45. Et son aide Paul Tra bi de Koifla.

46. Le conte de Bueti-bi-Dje qui explique pourquoi l'athérure se tient sur une patte donne bien le rat-toto et l'athérure comme fils du porc-épic, mais de mères différentes. L'athérure est fils de la première épouse, qui est zé le pangolin géant, Smutsia giganta tandis que le rat-toto a pour mère la seconde épouse, vole, le Céphalophe de Grimm, Sylivicapra grimmia, de pelage proche.

47. Pour tous les auteurs rapportant les propos tenus à ce sujet.

48. $m \mathcal{E}$-pó, "genoux de poulet » ou gù-lèè-bla, « la plante que gù tient en main », Leea guineensis (Vitaceae)

49. bowolo, Trichilia prieureana (Meliaceae).

50. dớ, Francolin, Francolinus bicalcaratus.

51. cle le zable, le zable de l'aigle huppard encore nommé gত্ّg $\alpha$ « quelque chose de suspendu » à cause de sa huppe, [ou zowùli br cle Aigle-fils du plus puissant parmi les oiseaux qui tuent les autres] zowùlì bl $c l \varepsilon$, Aigle huppard, Lophaetus occipitalis.

52. Vla, Daman, Dendrohyrax arboreus.

\section{RÉSUMÉS}

Les récits de l'origine de la plus belle et plus complète expression de l'art gouro, celle du masque Zamble et de sa mascarade, allient poésie des contes et précision du savoir.

Prenant au mot les surnoms animaux donnés par les Gouro de Zuenoula aux masques de la famille Zamble, soit Zamble lui-même, son frère sauvage Zàùlì et leur femme Gù, nous montrerons comment les mœurs attribuées respectivement à la panthère, au rat-toto et à la nandinie, savoir encyclopédique sur lequel s'appuient les contes, éclaire l'origine guerrière de ces cultes. Ces masques sacrés s'actualisant en mascarades publiques, les femmes à qui la connaissance du sacré reste interdite peuvent néanmoins en approcher le sens en suivant les analogies naturalistes que déploient chants et contes. À l'ethnologue, femme, d'acquérir cette connaissance pour saisir ces bribes de secret (sa sécrétion, secretas au sens de Zempleni, 1996).

The origin of the most beautiful and most complete expression of the Guro art, that of the Zamble mask and its masquerade, combine poetry of tales and precision of encyclopedic knowledge. 
Taking at its words the animal nicknames given by the Guro of Zuenoula to the masks of the Zamble family, namely Zamble himself, his wild brother Zàùli and their wife Gù, we show how the habits attributed respectively to the panther, to the Gambian pouched rat and to the African palm civet, encyclopedic knowledge on which the tales are based, illuminates the warlike origin of these cults. These sacred masks being actualized in public masquerades, women whose knowledge of the sacred remains forbidden can nevertheless approach its meaning by following the naturalistic analogies that are unveiled by songs and tales. It is up to the ethnologist, woman, to acquire this knowledge to seize these bits of secrecy (its secretions, secretas in the sense of Zempleni, 1996).

\section{INDEX}

Mots-clés : Gouro, Côte d'Ivoire, masques Zamble, secret, savoir naturaliste, pensée analogique, contes animaux, guerre

Keywords : Guro Ivory Coast, Zamble masks, secret, naturalist knowledge, analogic thinking, animal tales, war

\section{AUTEUR}

\section{CLAUDIE HAXAIRE}

Maître de Conférences à l'UFR de Médecine de l'Université de Bretagne Occidentale (UBO, Brest) CERMES (Centre de Recherche Médecine, Sciences, Santé, Santé Mentale et Société, Université Paris Descartes, EHESS, CNRS UMR 8211, INSERM U 988) 